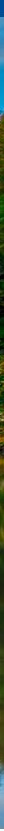

\title{
Characterizing Acoustic Signals in the Buildup to a Magnet Quench
}

Sujay Kazi

WG Diagnostics Meeting

7 August 2020

This manuscript has been authored by Fermi Research Alliance, LLC under Contract No. DE-AC02-07CH11359 with the U.S. Department of Energy, Office of Science, Office of High Energy Physics. 


\section{Introduction and objective}

- Superconducting magnets are used at world's leading particle accelerators to guide particles in circular trajectories

- Quench = loss of superconductivity

- Repeated quenches expensive b/c can damage magnet, require dumping liquid helium

- Buildup to quench not well understood

- This project aimed to investigate whether there is relationship between acoustic event energies and theoretical construct of minimum quench energy (MQE) 


\section{Magnet and sensor setup}

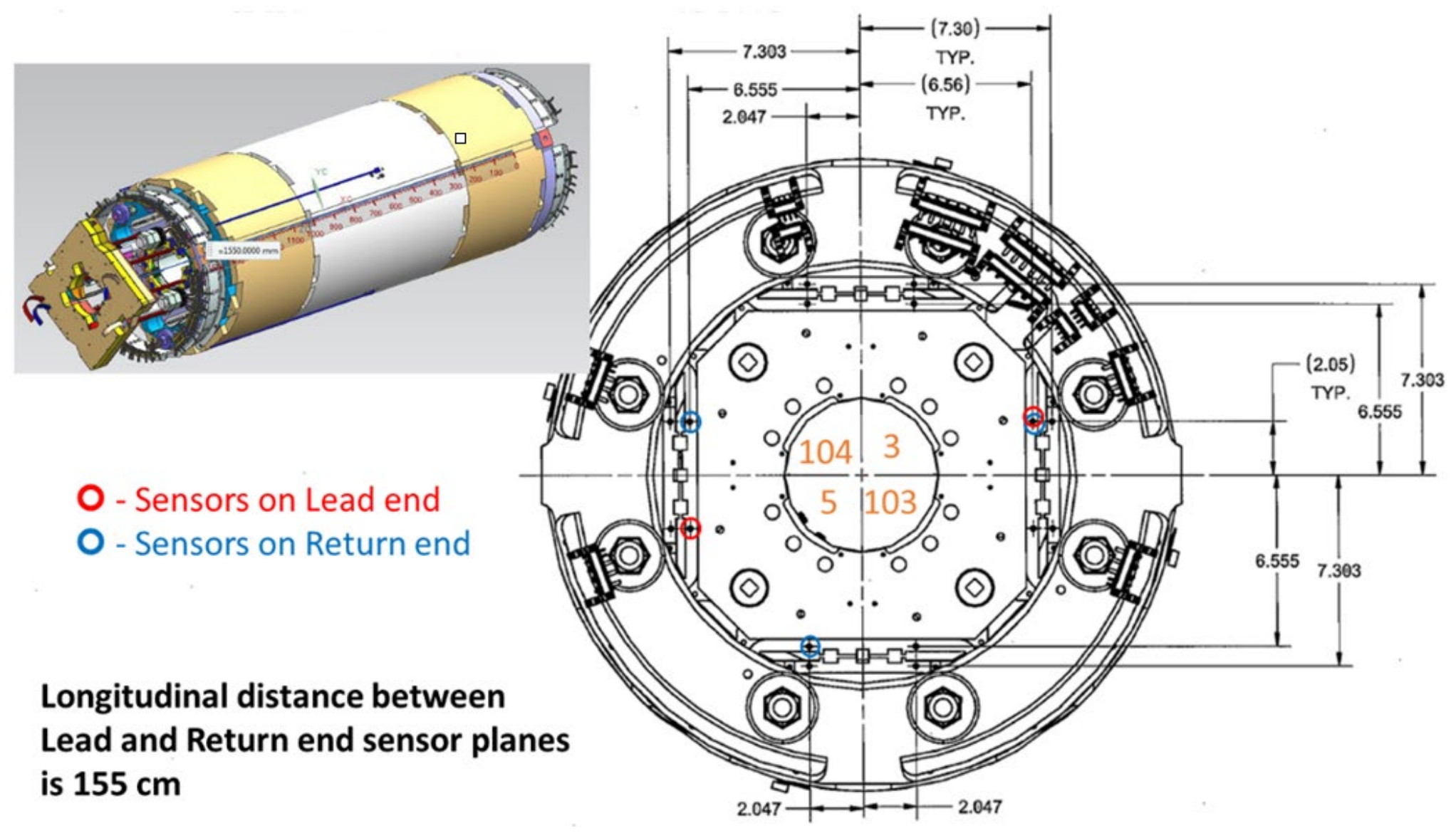

ai2, ai4 on lead end

ai0, ai1, ai3 on return end 


\section{Critical surface calculations}

$$
\begin{gathered}
J_{c}(B, T)=C B^{-1 / 2}\left(1-t^{2}\right)^{2} b^{-1 / 2}(1-b)^{2} \\
B_{c 2}(T)=\kappa(T) \alpha T_{c 0}\left(1-t^{2}\right) \\
\frac{\kappa(T)}{\kappa(0)}=\left[1-0.31 t^{2}(1-1.77 \ln t)\right]
\end{gathered}
$$

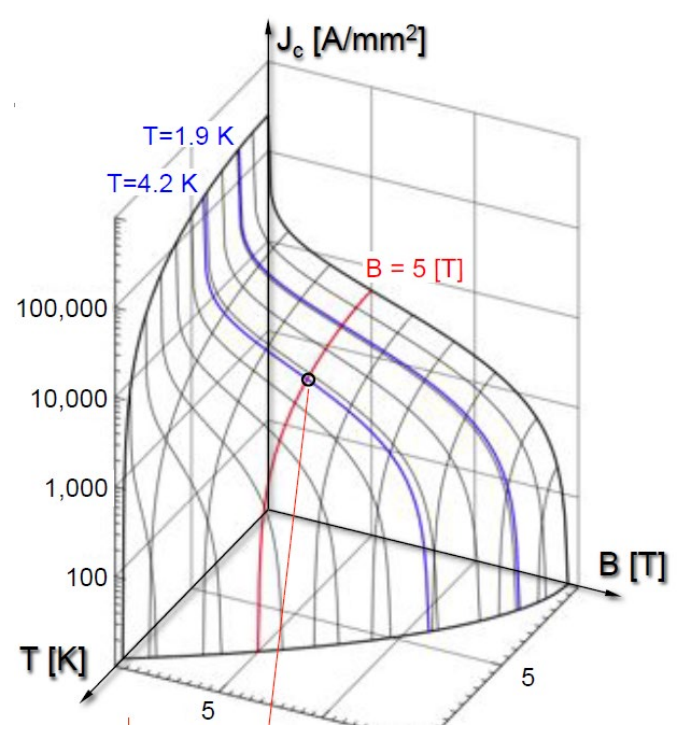

kappa = Ginzburg-Landau parameter

Sources: Summers et al. (1991), Wilson (2008) 


\section{Fitting to find $\mathrm{C}$ and alpha}

$$
\begin{aligned}
& C=9.56 \times 10^{9} \\
& \alpha=2.08
\end{aligned}
$$

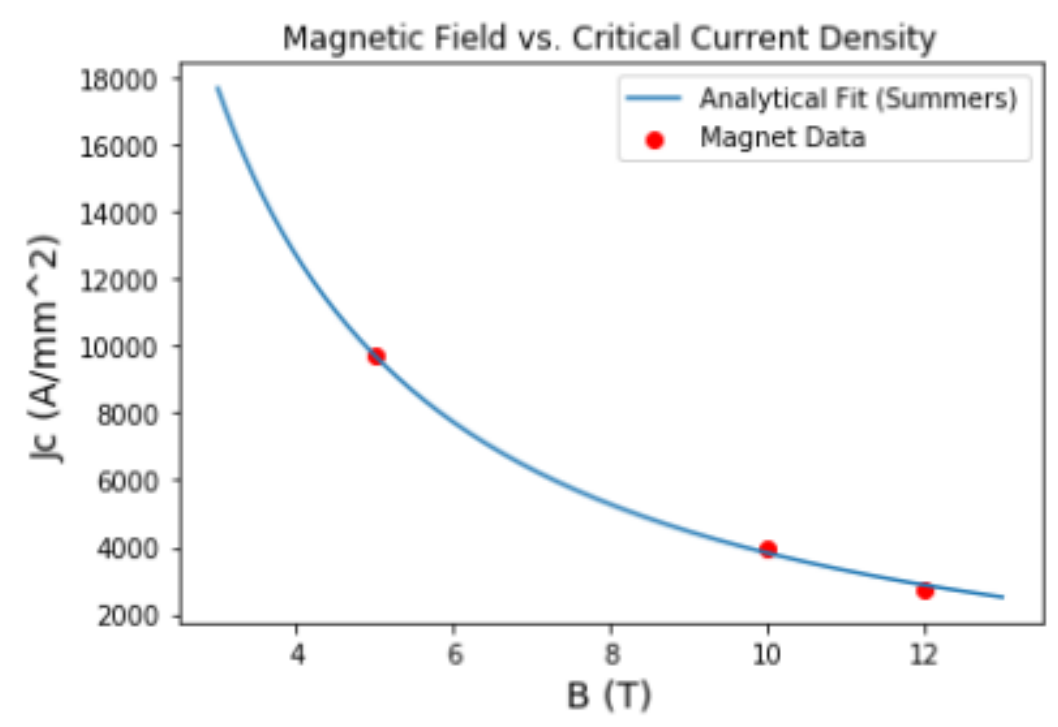

Covariance matrix:

$$
\left[\begin{array}{cc}
8.66 \times 10^{17} & -2.32 \times 10^{8} \\
-2.32 \times 10^{8} & 6.40 \times 10^{-2}
\end{array}\right] \quad \frac{\operatorname{Cov}(C, \alpha)}{\sqrt{\operatorname{Var}(C) \operatorname{Var}(\alpha)}} \approx-0.986
$$




\section{Length of MPZ and specific heat integral}

$$
\begin{aligned}
l_{0 W} & =\sqrt{\frac{2 A^{2} \lambda\left(T_{c}(I)-T_{o p}\right)}{\rho_{\mathrm{norm}} I^{2}}} \\
\epsilon_{I} & =V_{\mathrm{mpz}} \int_{T_{o p}}^{T_{c}} C_{p}(T) d T
\end{aligned}
$$

lambda $=$ thermal conductivity, rho_norm $=$ resistivity, $A=$ cross-sectional area Source: Stenvall et al. (2008) 


\section{Computing the energy of an acoustic event}

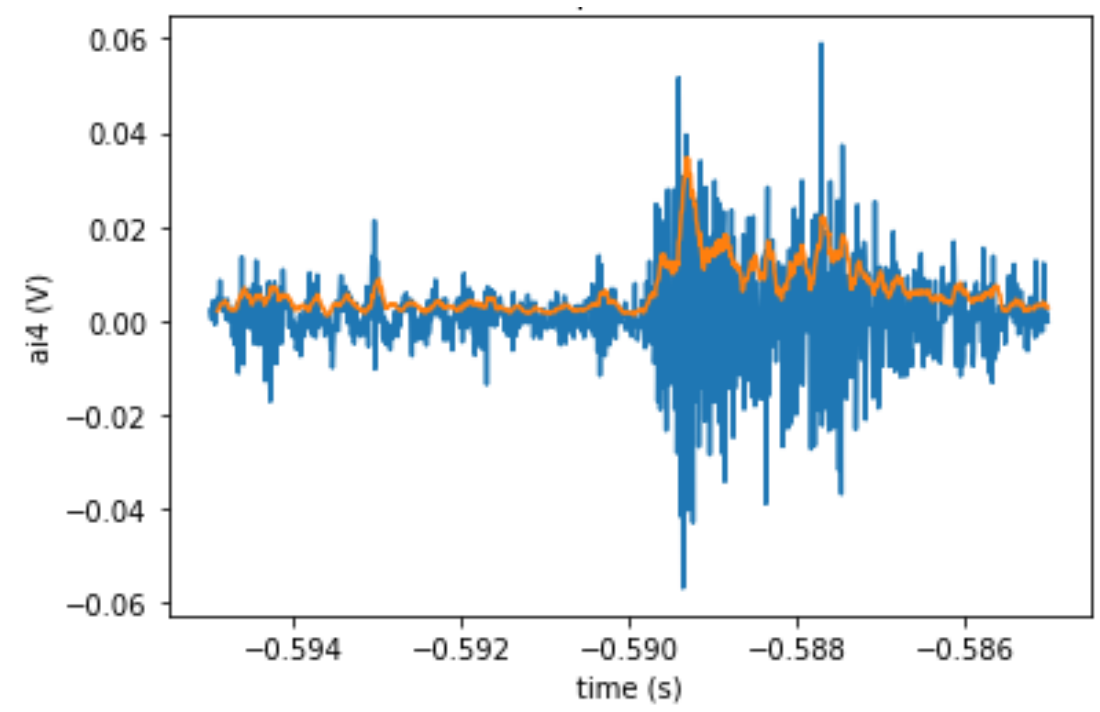

To compute the energy of the signal:

1. compute a rolling RMS with a window size of 10 frames $(0.1 \mathrm{~ms})$

2. take the square of the maximum

3. repeat for all sensors, take highest value

This is consistent with the fact that the energy of a wave is proportional to the square of its amplitude, and it avoids thorny issues regarding the exact start and end of an event. 


\section{Instances of strong trend}
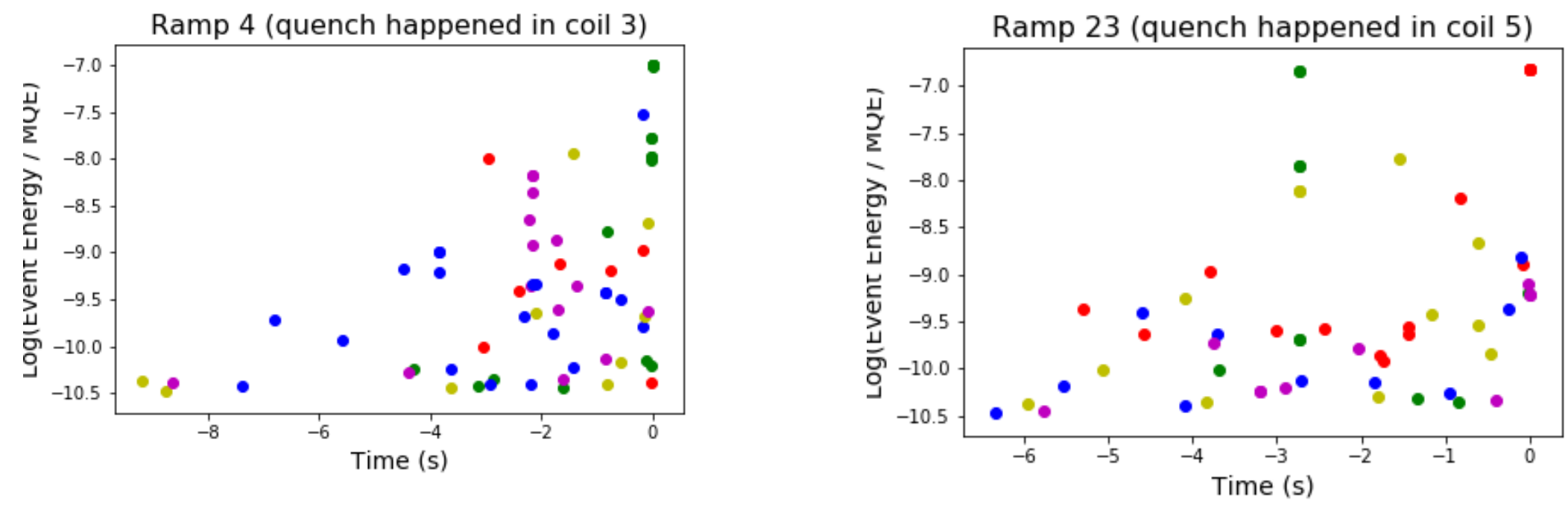

ai0 $=$ red, ai1 $=$ yellow, ai2 = green, ai3 = blue, ai4 = magenta Even in cases of strong trend, there were frequently outlier events, and there was no generally consistent threshold. 


\section{Instances of no trend or too few events}
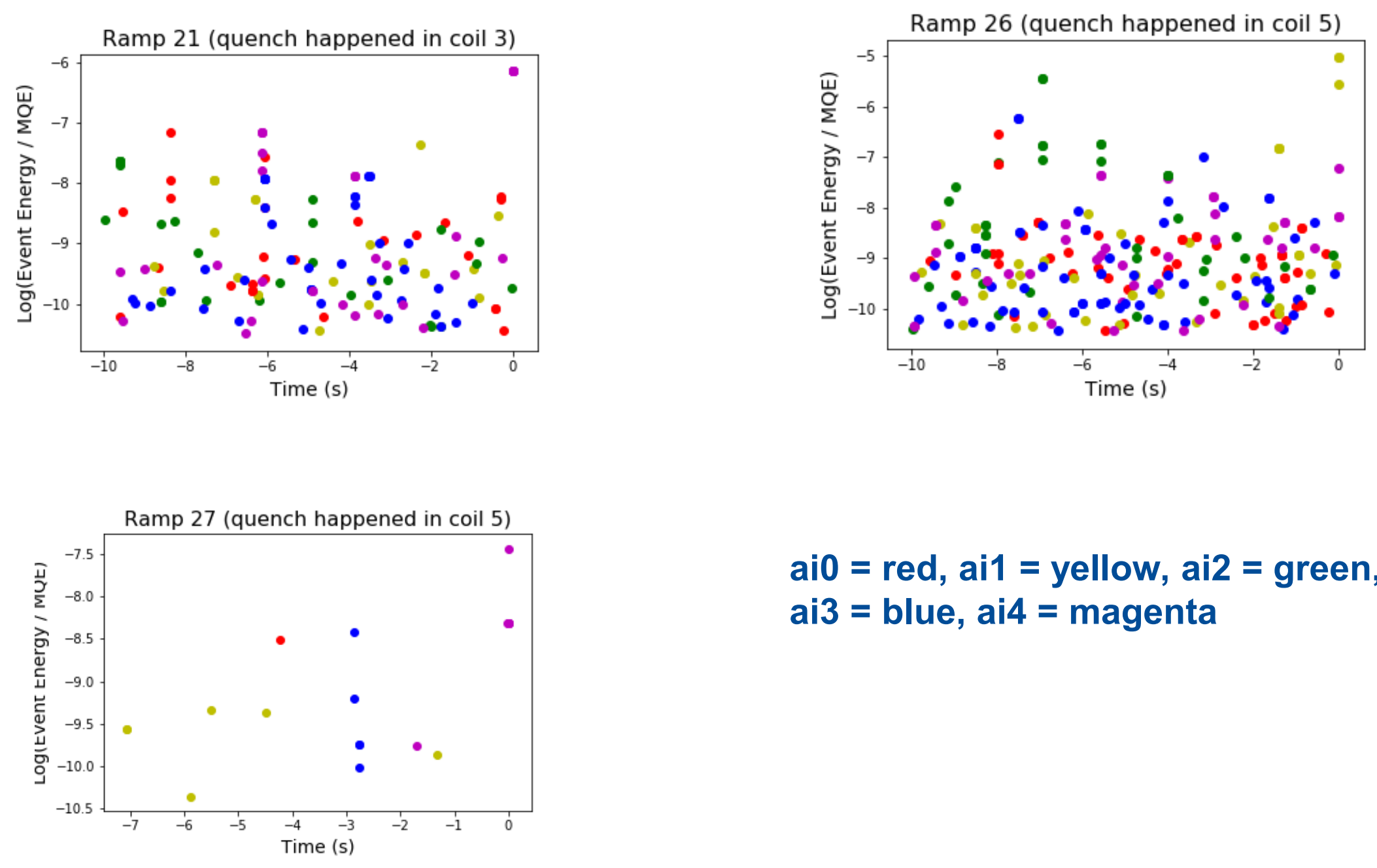

$$
\begin{aligned}
& \text { ai } 0=\text { red, ai } 1=\text { yellow, ai } 2=\text { green, } \\
& \text { ai3 }=\text { blue, ai } 4=\text { magenta }
\end{aligned}
$$




\section{Dependence of acoustic event energy on location not nearly as reliable as previously thought}
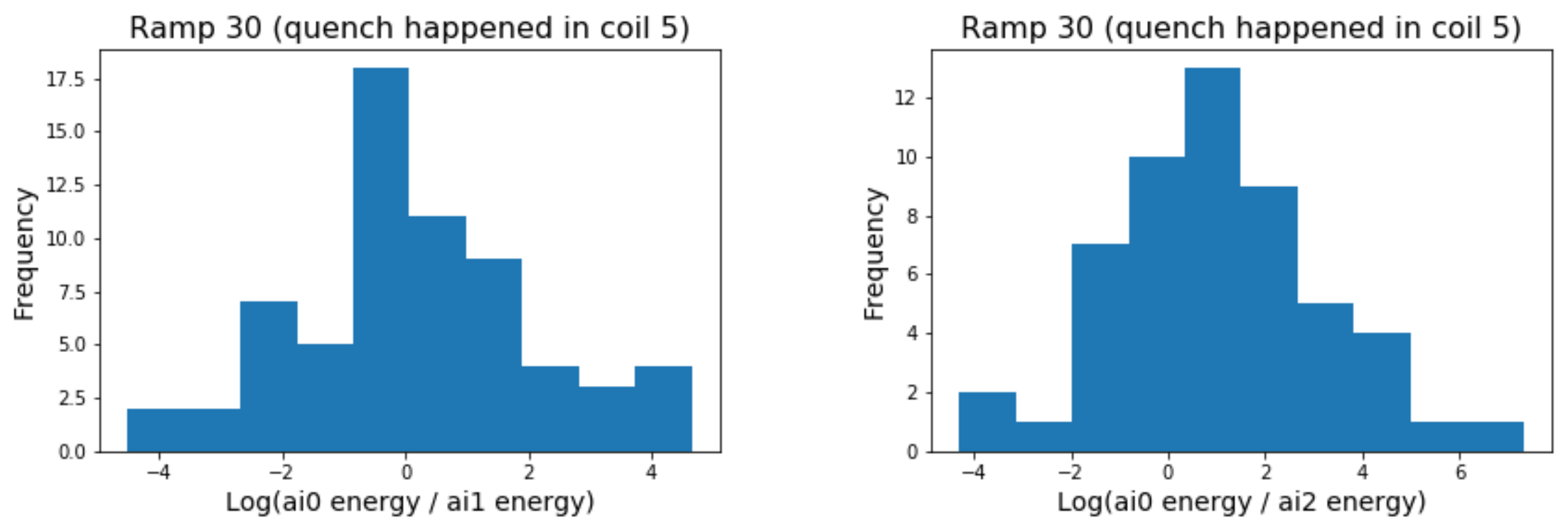

If the distance from an event origin to the sensor reliably predicted the event energy in that sensor, then the distribution on the left (where the sensors are on the same side) should be much narrower than the one on the right (where the sensors are on opposite sides). 


\section{Inconsistencies in arrival times}

ramp $=" 25 "$
sensor_list = ["ai0", "ai1", "ai2", "ai3", "ai4"]

for sensor in sensor,list:

plot_quench_event (ramp, sensor)

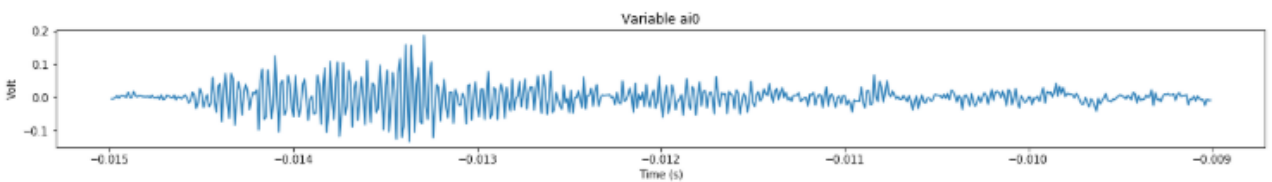

Flushing input history

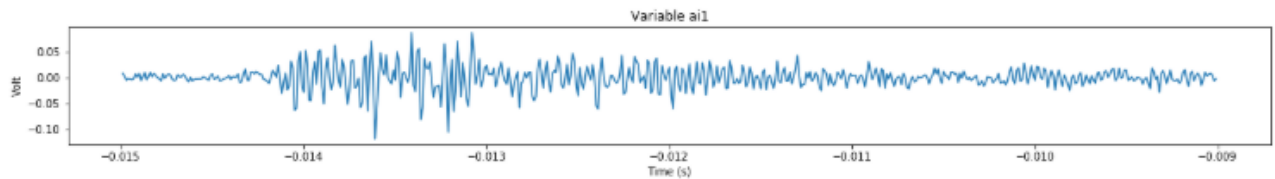

Flushing input history

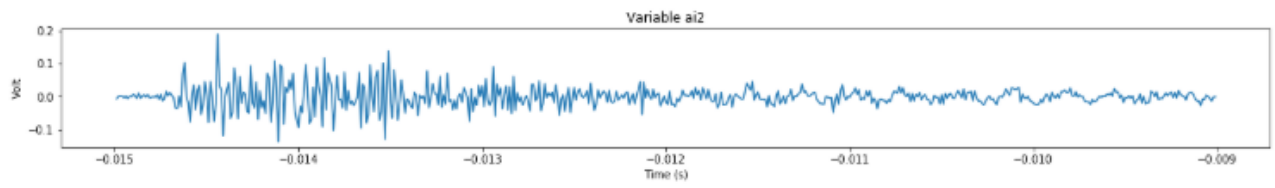

Notice how the start of the event in ai0 is much more closely aligned with the start of the event in ai2 and ai4, rather than with ai1 and ai3.

Flushing input history

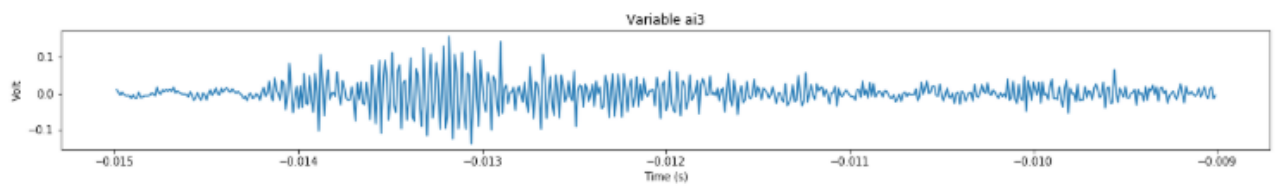

Flushing input history

input history

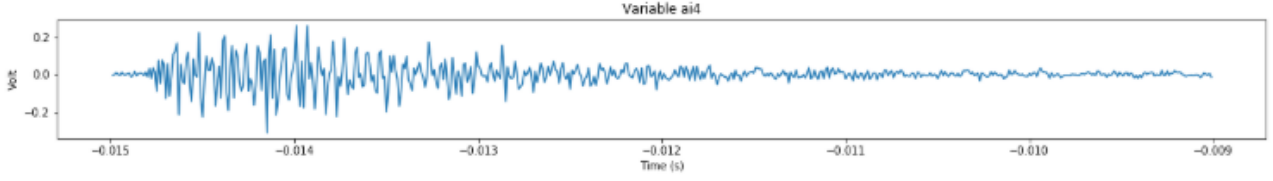

We were only able to find two prominent instances of this. However, given that these were quench events and have very clear acoustic signatures, it is still concerning. 


\section{Conclusions}

- Acoustic signals are messier than we previously thought in terms of comparisons between sensors

- Data validity is a serious concern that needs to be addressed

- Noticeable trend of increasing energy ratio over time, but only in some ramps

- Outlier events deserve investigation

- Lack of consistent threshold does not bode well for prediction algorithm, but doesn't rule it out completely 


\section{The first priority is to obtain better data and verify the quality of that data}

- Desired improvements in quality of data

- Make sure ramps aren't missing

- Try to have sensors on sides of magnet, not just ends

- Higher sampling rate (e.g. $1 \mathrm{MHz}$ ) for location detection

- Make sure that there aren't any sensor inconsistencies like the ones from the Ramp 23 and 25 quench events

- Try to understand why event can show up with such different strengths in different sensors, even ones on same side

- Real-time calibration of data: enter known pulse, observe how sensors respond

- Until resolved, analysis of event strengths very unreliable 


\section{Future avenues of exploration, once we know we have better data}

- Try to find more reliable way of computing "energy" of acoustic event

- Analyze "outlier" events: why didn't they trigger the quench earlier?

- Advanced location detection (cross-sectional location, not just longitudinal)

- Really dig into nature of individual events, try to understand them from physics perspective (e.g. through physical modeling of system)

- Try to turn general increasing trend of event energy as fraction of MQE into prediction algorithm

- Hunch is that ML would still be a lot better for prediction 


\section{Thank you for listening!}

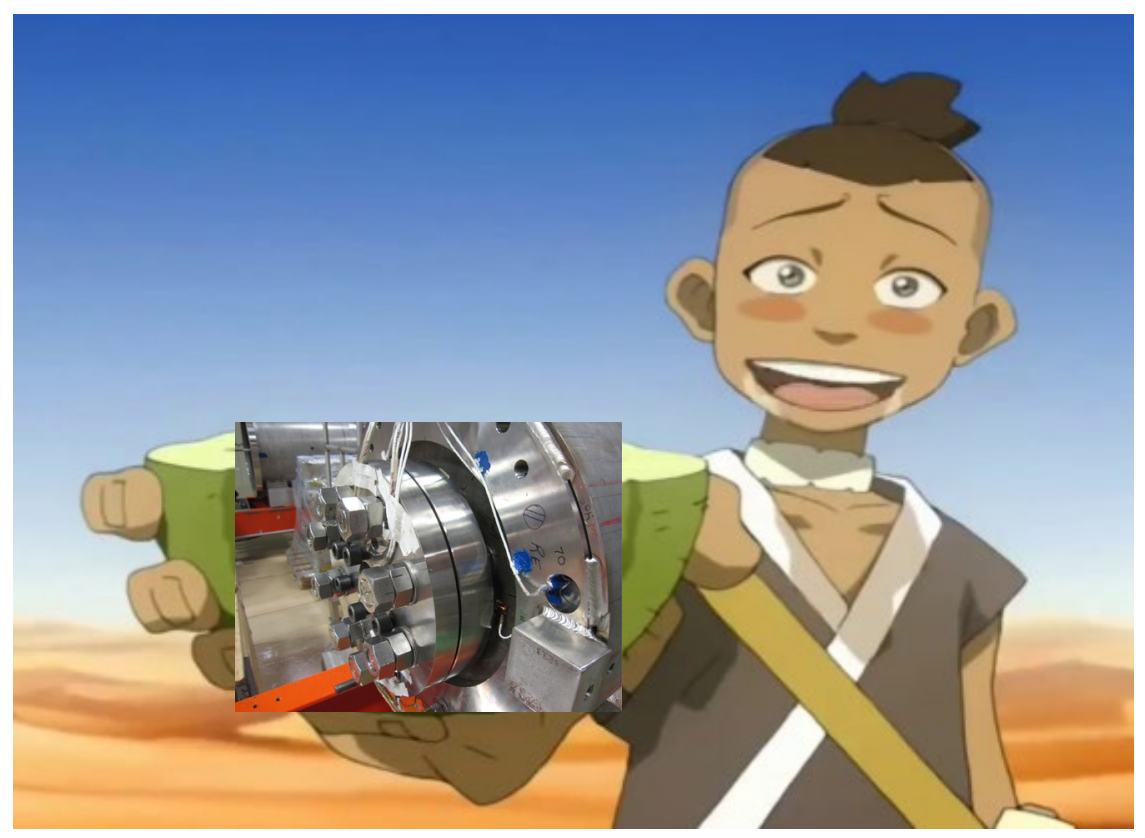

"It's the quenchiest!"

\section{Any questions?}

